\title{
ASPECTOS RELACIONADOS À PESCA ARTESANAL DO RIO CURIAÚ E LAGO TAPERA, MACAPÁ-AP
}

Marilu Teixeira Amaral ${ }^{1}$, Fredson Costa Rodrigues ${ }^{2}$, Marlen Cristina Teixeira Amaral $^{3}$, Orival de Deus Leite Júnior ${ }^{4}$

${ }^{1}$ Professora Mestra em Biologia Ambiental, Universidade do Estado do Amapá (mariamaral0824@gmail.com) Macapá-Brasil

${ }^{2}$ Pesquisador, Bacharel em Engenharia de Pesca, Universidade do Estado do Amapá, Macapá-Brasil

${ }^{3}$ Pesquisadora, Pós-graduanda em Desenvolvimento econômico e regional do Centro de Ensino Superior do Amapá, Macapá - Brasil

${ }^{4}$ Pesquisador, Bacharel em Engenharia de Pesca, Universidade do Estado do Amapá, Macapá-Brasil

Recebido em: 08/09/2015 - Aprovado em: 14/11/2015 - Publicado em: 01/12/2015 DOI: http://dx.doi.org/10.18677/Enciclopedia_Biosfera_2015_005

\begin{abstract}
RESUMO
A pesca desenvolvida na Amazônia possui grande importância para o desenvolvimento econômico regional, pois esta atividade fornece alimento, emprego e renda para as populações ribeirinhas. Diante do exposto, o presente trabalho teve por objetivo levantar junto aos pescadores artesanais das comunidades localizadas na área de proteção ambiental (APA) do rio Curiaú no município de Macapá, os aspectos relativos à caracterização pesqueira, suas diversidades culturais, aspectos socioeconômicos, os instrumentos usados para a prática da pesca, bem como verificar as espécies mais capturadas. Os dados qualitativos e quantitativos foram coletados entre outubro de 2014 e fevereiro de 2015, em visitas mensais a duas comunidades localizadas no quilombo do Curiaú (vilas do Curiaú de Fora e Curiaú de Dentro), pertencentes ao Município de Macapá (AP), pois estas duas localidades, dentre as comunidades do Quilombo, foram as que citaram o Lago Tapera e o Rio Curiaú como principais pesqueiros. A pesca na APA é de característica essencialmente artesanal, mesmo que grande parte dos pescadores da APA disponham de outra fonte de renda, a pesca apresenta papel importante no aspecto social, econômico e cultural. Casco e canoa são as principais embarcações utilizadas na pesca, no Curiaú de Fora $71 \%$ são proprietários de embarcações, sendo que no Curiaú de dentro somente $47 \%$ possuem barcos. O apetrecho de pesca mais utilizado é a malhadeira, e o tucunaré (Cichla sp.) foi a espécie descrita como a mais capturada e possui grande importância comercial para a população local.
\end{abstract}

PALAVRAS-CHAVE: Etnoconhecimento - Recursos pesqueiros - Sócio economia 


\title{
ASPECTS RELATED TO ARTISAN FISHING OF CURIAÚ RIVER AND TAPERA LAKE, MACAPÁ-AP
}

\begin{abstract}
The fishing in the Amazon has great importance for regional economic development, as this activity provides food, employment and income for coastal communities. This study aimed to present the main characteristics about artisanal fisheries communities located in the environmental protection area (APA) of Curiaú river in the city of Macapa, the aspects related to the fishing characterization, its cultural diversity, socioeconomic aspects, instruments used for the performance of fisheries, and may verify the species caught. Qualitative and quantitative data were collected between October 2014 and February 2015, monthly visits to two communities located in the quilombo Curiaú (Curiaú de fora and Curiaú de dentro), belonging to the city of Macapa (AP), as these two locations, among the Quilombo communities, are those cited Tapera Lake and Curiaú River main fishing grounds. Fishing in APA is essentially handmade characteristic, even if much of the APA fishermen are provided with another source of income, fishing plays an important role in the social, economic and cultural aspects. Hull and canoe are the main vessels used in fishing, in Curiaú de fora $71 \%$ are owners of vessels, and in the Curiaú de dentro only $47 \%$ have boats. The most widely used fishing tackle is gillnets, and tucunaré (Cichla sp.) Was described as the species and has the most captured great commercial importance.
\end{abstract}

KEYWORDS : Ethnoknowledge - Fishery Resources - Socioeconomic

\section{INTRODUÇÃO}

A pesca desenvolvida na Amazônia possui importância para o desenvolvimento econômico regional, pois esta atividade fornece alimento, emprego e renda para as populações ribeirinhas. Na Área de Proteção Ambiental do Rio Curiaú, essa atividade é desempenhada por mais de $90 \%$ da população residente seja para fins de lazer, subsistência ou atividade comercial (LEITE-JUNIOR et al., 2015).

Assim como em outras localidades do estado, a pesca de pequeno porte está constituída principalmente de forma artesanal e utiliza mão de obra familiar na produção. Os aparelhos e métodos de pesca dependem das espécies capturadas. As técnicas de pesca podem ser das mais simples, como linha de mão com anzóis, até as mais expansivas como redes de emalhar. As principais artes de pesca empregadas por pescadores da região são o espinhel e a malhadeira ou rede de espera. As embarcações são de madeira e variam de $30 \mathrm{~kg}$ a uma tonelada. A maior parte de produção é comercializada no mercado local (SILVA \& SILVA, 2006).

A quantidade de espécies exploradas pela pesca artesanal no Estado do Amapá, de acordo com AMARAL et al., (2015) as espécies do gênero Cichla (tucunaré) são citadas pelos pescadores como as mais comumente capturadas na região no período do inverno, o tucunaré é uma espécie de importância comercial na região da APA do Curiaú.

No estudo de ciências pesqueiras é importante considerar múltiplos aspectos como biologia, economia, sociologia e até mesmo o conhecimento empírico adquirido pelos pescadores ao longo dos anos são importantes para analisar os resultados encontrados e discutir determinados padrões.

Os aspectos relacionados a pesca artesanal são melhor explorados quando analisados por múltiplos fatores e através dos diversos atores envolvidos. Estudos 
que abordam o conhecimento adquirido pelos pescadores levando em conta sua percepção são importantes no que se refere a gestão pesqueira (RAMIRES et al., 2007; NUNES et al., 2011; RAMIRES et al., 2012; LEITE-JUNIOR et al., 2015).

BROOK \& MCLACHLAN (2009), ressaltam que a compreensão do Conhecimento Ecológico do Local (CEL) das populações a respeito dos recursos dos quais dependem é importante na percepção sobre padrões e processos ecológicos no que tange o manejo de áreas protegidas e a conservação de espécies e ecossistemas. Assim, o CEL envolve mais que o conhecimento ecológico acumulado, inclui também os sistemas de regras sociais necessárias para gerir os recursos locais, que são transmitidos através das gerações pela cultura local (BERKES et al., 2000). No Brasil, é crescente a ocorrência de trabalhos que debatem e analisam o conhecimento ecológico sobre recursos pesqueiros, sócio economicos, apetrechos de pesca utilizados, embarcações utilizadas durante a pesca, dentre outros (RODRIGUES, 2015).

Neste sentido, a pesca na área do Quilombo do Curiaú realizada de forma artesanal por pescadores da comunidade do Curiaú, proporciona para as famílias uma fonte de renda e subsistência. Localizada próximo à área urbana da cidade de Macapá, a Área de Proteção Ambiental (APA) do rio Curiaú encontra-se sob forte influência de ações antrópicas, em específico à pesca predatória por parte dos próprios moradores do quilombo e pessoas que moram na cidade, motivos que levaram a criação da APA.

Diante do exposto, o presente trabalho teve por objetivo levantar junto aos pescadores artesanais das comunidades localizadas na área de proteção ambiental (APA) do rio Curiaú no município de Macapá, os aspectos relativos à caracterização pesqueira, suas diversidades culturais, aspectos socioeconômicos, os instrumentos usados para o desempenho da pesca, bem como verificar as espécies mais capturadas.

\section{ÁREA DE ESTUDO}

\section{MATERIAL E MÉTODOS}

A APA do Rio Curiaú foi criada em 15 de setembro de 1998 pela Lei Estadual no 043 que estabeleceu uma área de 21.676 hectares e deu nome da unidade. Atualmente encontram-se sobrepondo o território da APA, em diferentes extensões, quatro quilombos (Rosa, Curralinho, Matafome e Curiaú) reconhecidos pela fundação Palmares. Destes, somente o quilombo do Curiaú apresenta título emitido pelo Instituto Nacional de Colonização e Reforma Agrária, INCRA, e tem cerca de 90\% do território sobrepondo a APA (RODRIGUES, 2015).

A APA do Rio Curiaú é uma Unidade de Conservação com finalidade de proteger os aspectos culturais das comunidades locais e os recursos naturais existentes, podendo-se utilizar os mesmos de maneira sustentável (BRASIL, Lei $\mathrm{N}^{\circ}$ 9.985, de 18 de julho de 2000.) (Figura 1). 

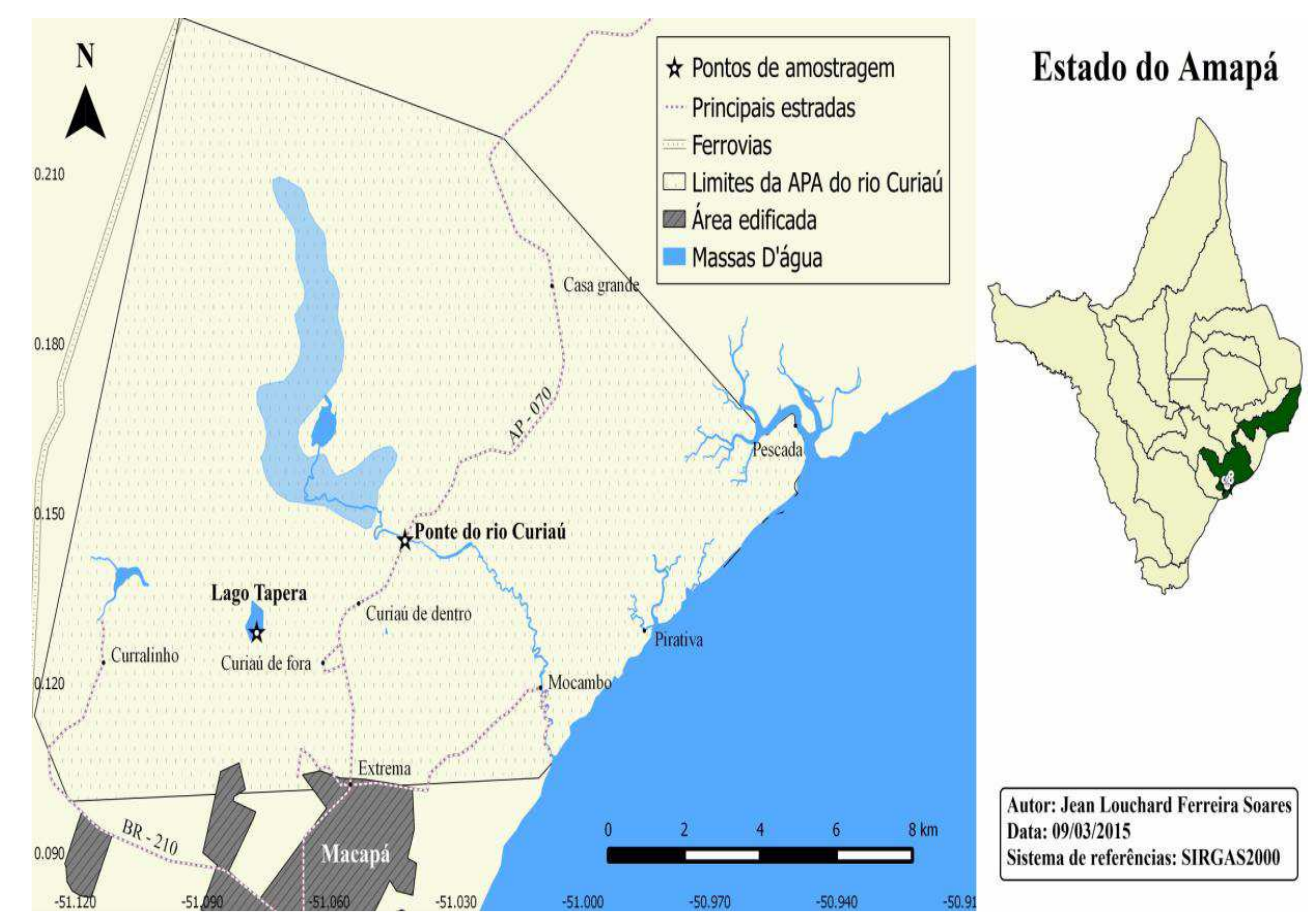

FIGURA 1 Localização do Rio Curiaú e lago Tapera, Macapá-AP

Fonte: RODRIGUES , (2015)

Esta área abrange ambientes de alta diversidade paisagística, oferecendo proteção à bacia do Rio Curiaú e às áreas de cerrado distribuídas na porção oeste da Unidade de Conservação (UC), envolvendo campos de várzea, matas de galeria e ilhas de matas, a leste essa vegetação é substituída por florestas de várzea (LEITE-JUNIOR, 2014).

A distribuição das áreas presentes na APA está representada da seguinte forma: 9.632.32 hectares de cerrado, o que corresponde a 43,31\%; a floresta de várzea ocupa $4.632,71$ hectares $(20,83 \%)$ e encontra-se distribuída numa faixa de orla fluvial de aproximadamente $5 \mathrm{~km}$ de largura; no eixo sul/nordeste aparecem os campos inundáveis ou campos de várzea correspondendo a 5.007,93 hectares $(22,51 \%)$; em seguida, aparece a mata de galeria com 1.369,59 hectares $(6,16 \%)$; as ilhas de matas, com $1.437,88$ hectares $(6,47 \%)$ e os rios e lagos com 159,25 hectares $(0,72 \%)$, números informados pelo Plano de Manejo da APA (CANTUÁRIA, 2011).

\section{COLETA DE DADOS SOCIOECONÔMICOS}

Os dados qualitativos e quantitativos foram coletados entre outubro de $2014 \mathrm{e}$ fevereiro de 2015, em visitas mensais as duas comunidades localizadas no quilombo do Curiaú (vilas do Curiaú de Fora e Curiaú de Dentro), pertencentes ao Município de Macapá (AP), pois estas duas localidades, dentre as comunidades do Quilombo, são as que citaram o Lago Tapera e o Rio Curiaú como principais pesqueiros.

No decorrer do ano de 2014 foram aplicados 28 questionários para levantamento de dados sobre o universo pesqueiro das comunidades, por meio da metodologia geradora de dados. Este método se baseou na aplicação de 44 perguntas abertas e 36 perguntas fechadas, visando obter o máximo de informações sobre o desenvolvimento da atividade pesqueira local. 
Também foi consultado o órgão responsável pela gestão da APA do rio Curiaú, a Secretaria de Estado do Meio Ambiente (SEMA-AP) sobre características da área e informações sobre a organização social de pescadores. A partir da estimativa do número de pescadores residentes na APA, incluindo os aposentados e os que estão em atividade foram selecionados para as entrevistas os pescadores com mais de 10 anos de experiência na atividade.

As entrevistas ocorreram nos locais nas respectivas comunidades do Curiaú de Fora e Curiaú de Dentro, inseridos na área de proteção ambiental APA rio Curiaú onde foram coletados dados gerais dos informantes como idade, estado civil, escolaridade, número de filhos, importância da pesca artesanal no orçamento familiar, relação com outras atividades econômicas, tempo de trabalho na pesca, locais utilizados para a pesca, métodos e aparelhos utilizados e espécies frequentemente capturadas e mais comercializadas.

\section{ANÁLISE DE DADOS}

O modelo da união de diversas competências individuais obtidos pelos dados de CEL e coleta de material biológico (NUNES et al., 2011) foi utilizado para a sistematização dos dados. Os dados obtidos foram tabulados em planilha eletrônica o que permitiu a construção dos gráficos e tabelas e análise estatística descritiva.

\section{RESULTADOS E DISCUSSÃO}

Aplicaram-se 28 questionários com pescadores artesanais mais experientes das comunidades do Curiaú de Fora e Curiaú de Dentro (Tabela 1).

TABELA 1- Número de entrevistados por comunidades pesqueiras da APA do rio Curiaú

\begin{tabular}{ccccc}
\hline Comunidades & Homens & Mulheres & Média de Idade & $\begin{array}{c}\text { Média de Anos } \\
\text { de atividade }\end{array}$ \\
\hline Curiaú de Fora & 13 & 0 & 48 anos & 34 anos \\
Curiaú de Dentro & 15 & 0 & 55 anos & 40 anos \\
Total & 28 & 0 & - & - \\
\hline
\end{tabular}

A faixa etária dos pescadores entrevistados na comunidade do Curiaú de Fora variou entre 20 a 80 anos, com média de 48 anos. No que se refere ao nível de escolaridade $61 \%$ dos pescadores entrevistados têm apenas o ensino fundamental incompleto. Não foi registrado nenhum pescador não alfabetizado na comunidade do Curiaú de Fora.

No que tange ao Estado de origem dos pescadores artesanais da APA do Rio Curiaú da comunidade do Curiaú de Fora, constatou-se que 100\% dos entrevistados são naturais do estado do Amapá. As amostras dos entrevistados em relação aos gêneros masculino e feminino adquirida neste estudo para esta comunidade foram compostas em sua totalidade por homens.

Em relação à outra atividade além da pesca praticada pelos pescadores artesanais da APA do Rio Curiaú da comunidade do Curiaú de Fora, para geração de renda os dados demostram que $31 \%$ dos entrevistados têm na construção civil fonte de renda complementar, $23 \%$ dos entrevistados são servidores públicos, $16 \%$ dos entrevistados são aposentados, ambos com 15\% têm na Segurança Privada e ENCICLOPÉDIA BIOSFERA, Centro Científico Conhecer - Goiânia, v.11 n.22; p.2856 2015 
no extrativismo outras fontes de renda, vale ressaltar que a pesca não é a principal atividade geradora de renda, entretanto apresenta significativa importância para os moradores da comunidade do Curiaú de Fora. No que diz respeito aos anos trabalhados na pesca os resultados obtidos mostram uma variação de 5 a 65 anos.

Foram realizadas 15 entrevistas diretas com aplicação de questionários semiestruturados com os pescadores da comunidade do Curiaú de Dentro. Os entrevistados apresentaram uma amplitude de idade que variou de 20 a 85 anos, com média de 55 anos. No que se referem ao nível de escolaridade $53 \%$ dos pescadores têm apenas o ensino fundamental incompleto.

Em relação a outras atividades, além da pesca, praticada pelos pescadores da comunidade do Curiaú de Dentro para geração de renda, os dados demostram que $46 \%$ dos entrevistados têm a agricultura como outra atividade, $23 \%$ trabalham na segurança privada, $15 \%$ são aposentados, ambos com $8 \%$ trabalham na construção civil e no funcionalismo público. No que diz respeito aos anos trabalhados, os resultados obtidos variam entre 12 a 70 anos na atividade.

Os pescadores com idade mais avançada foram encontrados nas comunidades do Curiaú de Fora (mais de 80 anos). RAMIRES et al., (2012) encontraram entre 39 a 56 anos para pescadores de comunidades pesqueiras do litoral sul de São Paulo, o que evidencia que na área de estudo os pescadores ingressam na atividade mais cedo que na região sudeste do Brasil, isso pode ocorrer devido a diversas falhas em políticas públicas do estado que priorizem a inserção de crianças em outras atividades que visem formação profissional.

No que se refere a escolaridade semelhante resultado encontrado neste trabalho foi registrado por SANTOS-FILHO (2010), que constatou que $78 \%$ dos pescadores artesanais na Vila do Sucurijú-AP possuem o ensino fundamental incompleto.

CABRAL (2001) em estudos realizados no rio Timbó - PE, encontrou pescadores com mais de 80 anos participando ativamente das atividades pesqueiras. RAMIRES et al., (2012) encontraram pescadores com mais de 65 anos de carreira, enquanto que neste trabalho foram encontrados trabalhadores com 70 anos de atuação na atividade pesqueira, o que configura um significante indicador da importância da APA do Curiaú para essas comunidades tradicionais.

De acordo com o que foi observado em campo e registrado em entrevistas foram encontrados dois tipos de embarcações para as comunidades do Curiaú de Fora e Curiaú de Dentro: casco e canoa. As informações atribuídas a cada uma das comunidades visitadas estão sumarizadas na Tabela 2.

TABELA 2 Descrição das embarcações por comunidades pesqueiras da APA do Curiaú

\begin{tabular}{lccccccc}
\hline Comunidade & Proprietário & \multicolumn{2}{c}{ Descrição } & Comp. & $\begin{array}{c}\text { Tipo de } \\
\text { propulsão }\end{array}$ & Capacidade & Tripulantes \\
\hline $\begin{array}{c}\text { Curiaú de } \\
\text { Fora }\end{array}$ & $77 \%$ & Casco & $\begin{array}{r}25 \\
\%\end{array}$ & Até $2,5 \mathrm{~m}$ & $\begin{array}{c}\text { Remo e } \\
\text { Mará }\end{array}$ & $<20 \mathrm{~kg}$ & 1 \\
\cline { 2 - 7 } & & Canoa & $\begin{array}{c}75 \\
\%\end{array}$ & $3 \mathrm{~m}$ & $\begin{array}{c}\text { Remo e } \\
\text { Mará }\end{array}$ & $<20 \mathrm{~kg}$ & 3 \\
\hline $\begin{array}{c}\text { Curiaù de } \\
\text { Dentro }\end{array}$ & $47 \%$ & Casco & $\begin{array}{c}29 \\
\%\end{array}$ & Até 3m & $\begin{array}{c}\text { Remo e } \\
\text { Mará }\end{array}$ & $<20 \mathrm{~kg}$ & 3 \\
\cline { 2 - 7 } & & Canoa & $\begin{array}{c}71 \\
\%\end{array}$ & 2,5 a 3m & $\begin{array}{c}\text { Remo e } \\
\text { Mará }\end{array}$ & $<20 \mathrm{~kg}$ & 2 \\
\hline
\end{tabular}


Na comunidade do Curiaú de fora $77 \%$ dos entrevistados são proprietários de embarcações, deste montante $25 \%$ são categorizados como sendo "casco" e $75 \%$ são denominados canoa. Casco é a embarcação cavada em um tronco de madeira e não possui quilha e a canoa é constituída de três tábuas e possui quilha, ambas as embarcações utilizam o remo ou o mará como modo de propulsão.

Assim como no Curiaú de Fora, a comunidade do Curiaú de Dentro apresenta apenas duas categorias de embarcação (Casco e Canoa), sendo que para o Curiaú de Dentro somente $47 \%$ dos entrevistados são donos de embarcações. ALBUQUERQUE \& BARTHEM (2008) citam que os pescadores empregam canoas ou casco para as pescarias comerciais, individuais ou em grupos, do tamoatá nos rios e lagos da região do alto Arari.

Neste trabalho os apetrechos de pesca mais utilizados e que foram registrados em todas as comunidades foram a malhadeira e o caniço (tabela 3). Nas comunidades do Curiaú de Fora $100 \%$ dos pescadores entrevistados relataram utilizar a malhadeira durante a captura. Na comunidade do Curiaú de Dentro o uso do caniço esteve $100 \%$ presente nas respostas dos entrevistados (Tabela 3 ).

TABELA 3 Apetrechos de pesca utilizados pelos pescadores das comunidades da APA do rio Curiaú

\begin{tabular}{ccc}
\hline Artes de pesca & Curiaú de Fora & Curiaú de Dentro \\
\hline Mallhadeira & $100 \%$ & $95 \%$ \\
\hline Caniço & $97 \%$ & $100 \%$ \\
\hline Linha de mão & $68 \%$ & $75 \%$ \\
\hline Tarrafa & $25 \%$ & $48 \%$ \\
\hline Arpão & $26 \%$ & $10 \%$ \\
\hline Zagaia & $70 \%$ & $45 \%$ \\
\hline Lanterna & $5 \%$ & $5 \%$ \\
\hline Farol e bateria & $10 \%$ & $0 \%$ \\
\hline Poronga & $0 \%$ & $5 \%$ \\
\hline Espinhel & $0 \%$ & $0 \%$ \\
\hline
\end{tabular}

A pesca com malhadeira é praticada com uma rede de extensão variada chegando até 150 metros, confeccionada de nylon, com altura que varia de 2 a $3 \mathrm{~m}$, com malha entre 5 e $10 \mathrm{~cm}$ entre nós opostos, ainda que o IBAMA estipule $7 \mathrm{~cm}$ no mínimo (GUTMAN, 2005). Esse apetrecho possui em um dos lados pequenas boias de isopor e no lado adverso pequenos pedaços de chumbo espalhados ao longo da dimensão, somando em média 6 kg (Figura 2). 


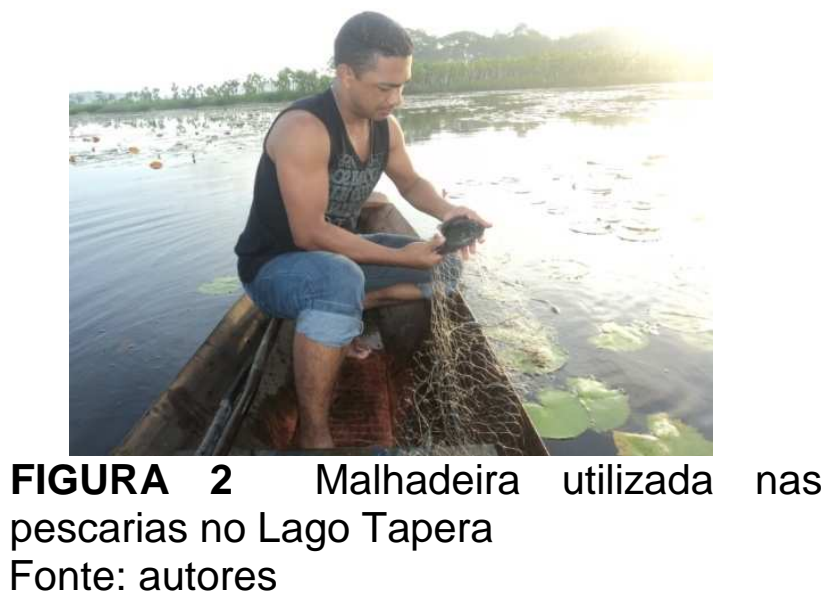

Foram relatadas 10 etnoespécies pelos pescadores do Lago Tapera e Rio Curiaú (Tabela 4). A espécie Tucunaré (Cichla sp) apresenta grande importância comercial para ambas comunidades.

TABELA 4 Porcentagem de etnoespécies mais capturadas e mais comercializadas nas comunidades da APA do rio Curiaú citados pelos pescadores do Curiaú de Fora e Curiaú de Dentro.

\begin{tabular}{cccc}
\hline Nome vernacular & Espécie & Curiaú de fora & Curiaú de Dentro \\
\hline Traíra & Hoplias malabaricus & $92,3 \%$ & $46,7 \%$ \\
\hline Tucunaré & Cichla sp. & $92,3 \%$ & $100 \%$ \\
\hline Mafurá & Pygopristis denticulata & $84,6 \%$ & $100 \%$ \\
\hline Tambaqui & Colossoma macropomum & $23,1 \%$ & $0 \%$ \\
\hline Piranha & Serrasalmus sp. & $69,2 \%$ & $100 \%$ \\
\hline Jeju & Hoplerythrinus Unitaeniatus & $61,5 \%$ & $100 \%$ \\
\hline Tamoatá & Hoplosternum littorale & $38,5 \%$ & $100 \%$ \\
\hline Apaiarí & Astronotus sp & $15 \%$ & $0 \%$ \\
\hline Acará & Cichlasoma sp. & $7,7 \%$ & $0 \%$ \\
\hline Sarapo & Gymnotus sp. & $7,7 \%$ & $0 \%$ \\
\hline
\end{tabular}

SANTOS et al., (2014) encontraram 22 etnoespécies para a localidade de Pracuúba-AP, distribuídas em 4 ordens e 12 famílias, a família Characidae apresentou o maior número de espécies, seguida de Erythrinidae e Cichlidae.

\section{CONCLUSÃO}

- A pesca desenvolvida na APA do rio Curiaú é essencialmente artesanal;

- A atividade pesqueira não é a única atividade geradora de renda para as comunidades estudadas, no entanto apresenta papel importante nos aspectos social, econômico e cultural na APA;

- O tucunaré (Cichla sp) e a traíra (Hoplias malabaricus) são importantes recursos pesqueiros capturados pelos pescadores da APA;

- Verificou-se a necessidade de estudos complementares referentes ao conhecimento da ictiofauna presente na APA;

- Faz-se necessário que estudos de monitoramento sejam feitos para que se eleve o conhecimento sobre os aspectos relativos à pesca na APA com o intuito de fazer uso dos recursos pesqueiros nas comunidades estudadas de maneira sustentável. 


\section{REFERÊNCIAS}

ALBUQUERQUE, A. A. de; BARTHEM, R. B. A pesca do tamoatá Hoplosternumlittorale (Hancock, 1828) (Siluriformes: Callichthyidae) na ilha de Marajó. Bol. Mus. Para. Emílio Goeldi, Ciências Humanas, Belém, v. 3, n. 3, p. 359-372, set./dez. 2008.

AMARAL, M. T; RODRIGUES, F. C.; LEITE-JUNIOR, O. D.; SOUZA, P. L.; ESTEVES, Y. A. Conhecimento Etnológico da ictiofauna no lago Tapera e Rio Curiaú, Macapá - Ap. CONGRESSO DE ENGENHARIA DE PESCA, 19., 2015, São Luiz, Maranhão, Anais... São Luiz, 2015.

BERKES, F.; COLDING, J.; FOLKE, C. Sacred Ecology: Traditional Ecological Knowledge and Resource Management. 2000.

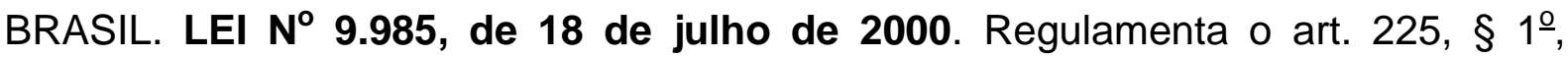
incisos I, II, III e VII da Constituição Federal, institui o Sistema Nacional de Unidades de Conservação da Natureza e dá outras providências, 2000.

BROOK, R. K.; MCLACHLAN, S. M. Trends and prospects for local knowledge In: ecological and conservation research and monitoring. Biodiversity Conservation 17, 3501-3512. 2009.

CABRAL, A. L. Aspectos sócio-culturais e implicações ambientais das formas de uso e ocupação do espaço estuarino do rio Timbó, Estado de Pernambuco, Brasil. 2001. Dissertação (Mestrado em Desenvolvimento em Meio Ambiente) Universidade Federal do Paraíba, João Pessoa, 2001.

CANTUÁRIA, E. R. APA do Rio Curiaú e a cidade de Macapá: relações sociais jurídicas e ambientais. Dissertação (mestrado em Direito Ambiental e Políticas Públicas). - Universidade Federal do Amapá, Macapá-AP, 2011.

GUTMAN, S. M. Caracterização do sistema de produção do lavrador-pescador em quinze comunidades rurais do entorno do Lago de Viana, na baixada Maranhense. 102 f. 2005. Dissertação (Mestrado em Agroecologia) - Universidade Estadual do Maranhão, Maranhão, 2005.

LEITE-JUNIOR, O. D. Caracterização da atividade pesqueira na Área de Proteção Ambiental do rio Curiaú municipio de Macapá, estado do Amapá. 2014. Monografia (Graduação em Engenharia de Pesca) - Universidade do Estado do Amapá, Macapá, 2014.

LEITE-JUNIOR, O. D; AMARAL, M. T.; APARÍCIO, G. K.; SOUZA, P. L. A pesca na Área de Proteção Ambiental do Curiaú, Macapá-AP, Brasil. CONGRESSO DE BIOLOGIA MARINHA, 5., 2015, Porto de Galinhas, Ipojuca - CE. Anais... Porto de Galinhas, 2015.

NUNES, D. M. HARTZ, S. M.; SILVANO, R. A. M. Conhecimento ecológico local e científico sobre os peixes na pesca artesanal no sul do Brasil. Bol. Inst. Pesca, 37(3): $209-223.2011$. 
RAMIRES, M.; MOLINA, S. M. G.; HANAZAKI, N. Etnoecologia caiçara: o conhecimento dos pescadores artesanais sobre aspectos ecológicos da pesca. Biotemas, 20: 101-113. 2007.

RAMIRES, M; BARRELLA, W. ; ESTEVES, A. M. Caracterização da Pesca Artesanal e o Conhecimento pesqueiro local no Vale do Ribeira e Litoral Sul de São Paulo. Revista ceciliana. V.4, n.1, p.37-43, 2012.

RODRIGUES, F. C. Conhecimento etnológico da ictiofauna no lago Tapera e rio Curiaú, Macapá-AP. 2015. Monografia (Graduação em Engenharia de Pesca) Universidade do Estado do Amapá, Macapá, 2015.

SANTOS-FILHO, A. P. Aspecto sócio econômico da atividade pesqueira na vila de sucurijú Amapá. 2010. Monografia (Graduação em Engenharia de Pesca) Universidade do Estado do Amapá, Macapá, 2010.

SANTOS, J.R.; SOUZA, L.P. de; LOBATO, A. dos S.; OLIVEIRA, N. I. da S. de; FLORENTINO, A.; LOBATO, A. do N.; CAVALCANTE, B. R. S. Etnoictiologia como subsídio ao defeso de espécies de peixes comerciais na Amazônia Oriental, Pracuúba, Amapá, Brasil. Revista de Ciências da Amazônia, Macapá, n. 2, v. 1, p. 1-12, 2014.

SILVA, L. M. A. da; SILVA, S. L. de F. A atividade pesqueira na região atlântica da costa do Amapá: Município de Amapá, Pracuúba, Tartarugalzinho e baixo Araguari. In: REDE Cooperativa de Monitoramento Ambiental de Áreas sob Influência da Industria Petrolífera. Natal: CT-PETRO, 2006. 